\title{
Skin Cancer Classification using Random Forest
}

\author{
S. Nandhini, Mohammed Abdul Sofiyan, Sushant Kumar, Adnan Afridi
}

\begin{abstract}
Skin cancer is a very big health issue in today's fastgrowing population not only for old age people but for all age groups. We are classifying skin cancer of a person according to dermatoscopic images into seven different types. We handle this issue utilizing the HAM10000 (Human-Against-Machine with 10000 training images) data-set. The finalized dataset includes 10001 dermatoscopic pictures which are released as a readiness set for academic machine learning purposes and are openly available through the ISIC archive. We are classifying skin cancer of a person according to dermatoscopic images into seven different types.Through this research a person will get to know that if he/she suffering from any kind of skin cancer or not, so before going to consult any doctor a person will have some assurance about skin cancer.
\end{abstract}

Keywords: skin cancer, dermatoscopic, HAM10000, ISIC archive

\section{INTRODUCTION}

The enthusiasm of the biomedical academic network for PC bolstered skin injury examination and portrayal has been expanded during the most recent years. Skin disease is among the most incessant kinds of malignant growth and one of the most dangerous tumors. Its frequency has expanded quicker than that of practically all different malignancies, and the yearly rates have expanded on the request for $3 \%-7 \%$ in reasonable cleaned populace in ongoing decades. As of now, somewhere in the range of 2 and 3 million non-melanoma skin malignant growths and 132000 melanoma skin tumors happen all around every year. In addition, the occurrence rates of skin malignancy have been expanding for as far back as century, it can be found in countries, for example, United Kingdom, where the growth of melanoma has extended $120 \%$ since the 90 's, or United States of America (from 27,000 cases in 1991 to 91,000 out of 2018). The explanation of this example lies not simply in the reduction of the ozone layer, which has decreased the protection from the Ultraviolet radiation, yet moreover on the harming prologue to the sun or the solarium and the use of tanning.

The medicinal network has contributed a great deal of time and cash in counteractive action crusades, raising the attention to the populace. In any case, changing reckless conduct may not ensure security, as the likelihood of getting skin disease likewise relies upon the quantity of burns from the sun that individuals got for an incredible duration. Subsequently, it is likewise essential to put resources into the improvement of advancements that can be utilized for early finding of skin malignancy. The fundamental plan issues for the best possible portrayal of skin injuries concern the picture securing, the picture handling and investigation, the component extraction, and the characterization technique.

Revised Manuscript Received on November 15, 2019.

* Correspondence Author

MS. S. Nandhini*, Assistant Professor (O.G) at SRM Institute of science and technology.

Mohammed Abdul Sofiyan, Department of B.tech in SRM Institute of science and technology.

Sushant Kumar, Department of B.tech in SRM Institute of science and technology. technology.

(C) The Authors. Published by Blue Eyes Intelligence Engineering and Sciences Publication (BEIESP). This is an open access article under the CC BY-NC-ND license (http://creativecommons.org/licenses/by-nc-nd/4.0/)
Adnan Afridi, Department of B.tech in SRM Institute of science and

Two of the real impediments of dermoscopy are its subjectivity and necessity of broad preparing. An extraordinary effort has been made by the exploration network in the improvement of Computer Aided Diagnosis apparatuses that can be utilized by dermatologists to defeat the previously mentioned problems. This system consists of following steps:
a) Image pre-processing
b) Lesion-segmentation
c) Feature-extraction
d) Feature-selection
e) Classification.

\section{A. IMAGE PREPROCESSING}

Image pre-processing is a significant advancement to manage pictures that don't have adequate quality to be inquired about. This nonappearance of huge worth can be an immediate consequence of the vicinity of old rarities (e.g., hair) that can oppositely influence the presentation of the resulting progresses. Dermoscopy pictures might be procured utilizing various contraptions and light conditions, rendering clashing covering data. Thusly, it might be essential to join a covering correction step in the pre-dealing with organize.

\section{B. LESION-SEGMENTATION}

Lesion-Segmentation is an inconvenient assignment that has been completely investigated in the organization. The amazing assortment of lesion shapes, sizes, and tints comparatively as various skin types and surfaces make it hard to build up a strong division figuring. A careful division is recognized to be basic to accomplish a genuine extraction of highlights and following lesion delineation. To evade potential misclassification in perspective on mistaken division, a few computer-aided design structures intertwine self-loader division.

\section{FEATURE-EXTRACTION}

Feature-extraction is a huge development to get a discriminative depiction of the skin wounds. Finding fitting features is a problematic system and a huge amount of research has been performed in this field, causing it possible to recognize an immense scope of features that to portray skin wounds.

\section{FEATURE-SELECTION}

Feature-selection is used to reduce the dimensionality of the feature space in a bit of the computer-aided design structures by discarding unnecessary, abundance, or noisy features.

\section{E. CLASSIFICATION}

Lesion classification is the last advance of the computeraided design framework. Here, a classification calculation is set up to envision an investigation.

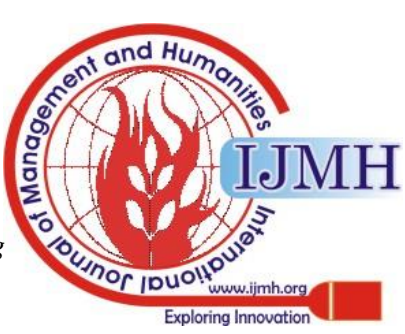




\section{Skin Cancer Classification using Random Forest}

Most by far of the computer-aided design framework base on the capability among melanoma and friendly or atypical nevi, as a result of significant level of danger related with the past kind of malignant development.

\section{RANDOM FOREST CLASSIFIER}

A sporadic wood multiway classifier involves different trees, with each tree created using some sort of randomization. The leaf center points of each tree are named by evaluations of the back spread over the image classes. Every inside center point contains a test that best parts the space of data to be described. An image is gathered by sending it down each tree and conglomerating the landed at leaf transports. Assertion can be mixed at two during planning: in subsampling the arrangement data with the objective that each tree is created using a substitute subset; and in picking the center point tests.

\section{RANDOM FOREST ALGORITHM}

Random Forest include following steps:

1. First picking preparing dataset. Use the bootstrap arbitrary inspecting strategy to recoup $\mathrm{K}$ preparing sets from the first dataset of $\mathrm{N}$ properties, with the size of each preparation set proportional to that of the first preparing dataset.

2. Amassing the Random Forest model. Make an arrangement relapse tree for the majority of the bootstrap arranging sets to pass on $\mathrm{K}$ decision trees to layout a "Forest" these trees are not pruned. Looking improvement of each tree, this methodology doesn't pick the best highlights as internal focus focuses for branches anyway rather the extending system is an arbitrary choice of $\mathrm{m} \leqslant \mathrm{M}$ everything being proportional.

3. Make clear casting a ballot. Since the readiness methodology of each choice tree is autonomous, the arrangement of the arbitrary backwoods can work in parallel, which basically improves productivity. The arbitrary woods can be worked by joining $\mathrm{K}$ choice trees prepared similarly. When ordering the information tests, the results depend upon the fundamental democratic of the yield of each choice tree. The Random Forest count chooses the examples by building a movement of free and passed on choice trees and chooses the last class of the model according to each choice tree.

The confusion matrix of skin cancer cancer classification:

True Positive Rate $=\mathrm{TP} /(\mathrm{TP}+\mathrm{FN})$.

Recall $=$ True Positive.

Positive Rate $=$ FP $/(\mathrm{FP}+\mathrm{TN})$.

Positive Predictive Value $=\mathrm{TP} /(\mathrm{TP}+\mathrm{FP})$.

Accuracy $=(\mathrm{TP}+\mathrm{TN}) /(\mathrm{FP}+\mathrm{TP}+\mathrm{TN}+\mathrm{FN})$.

Abbreviation:

$\mathrm{TP}=$ True-Positive

$\mathrm{FP}=$ False-Positive

$\mathrm{TN}=$ True-Negative

$\mathrm{FN}=$ False-Negative

$$
f i_{i}=\frac{\sum_{j: \text { node } j \text { splits on feature } i} n i_{j}}{\sum_{k \in \text { all nodes }} n i_{k}}
$$

Feature Ranking can be calculated by the formula:

$$
R F f i_{i}=\frac{\sum_{\text {jeall trees }} \text { norm } f i_{i j}}{T}
$$

The final feature, at the Random Forest level, is its average over all the trees. The sum of the feature's importance value on each tree is calculated and divided by the total number of trees:

General decision tree for random forest algorithm:

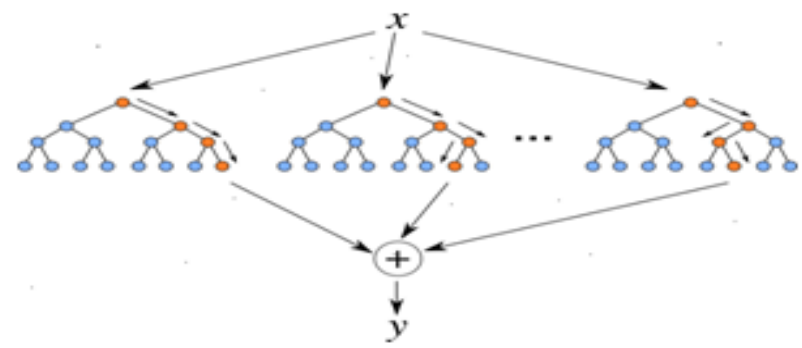

Fig. 1. General Decision Tree

Samples dermotoscopic images for the 7 different types of skin lesions:

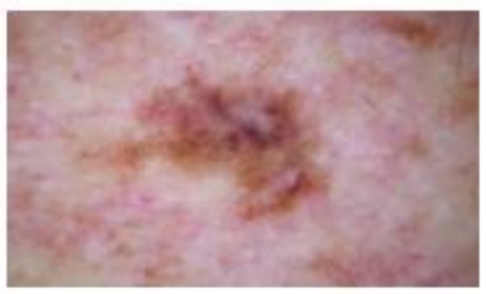

Fig. 2. Melanoma

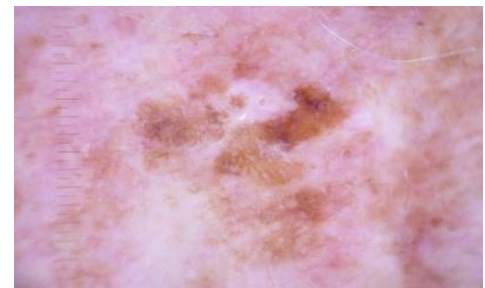

Fig. 3 Benign keratosis-like lesion

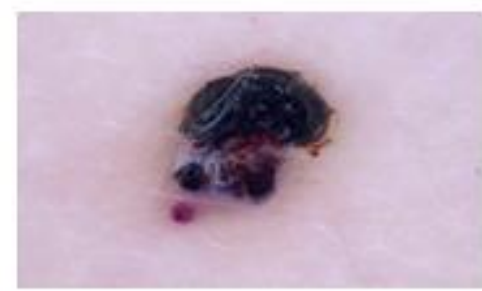

Fig. 4. Melanocytic nevi

Published By:

Blue Eyes Intelligence Engineering

\& Sciences Publication

(C) Copyright: All rights reserved. 


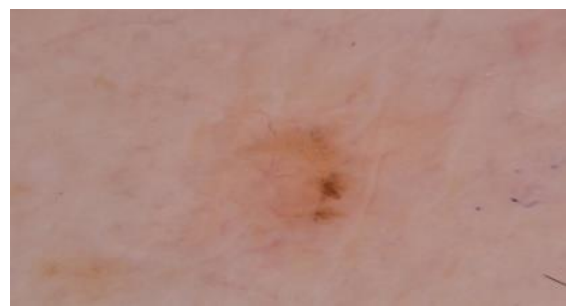

Fig. 5. Basal Cell Carcinoma

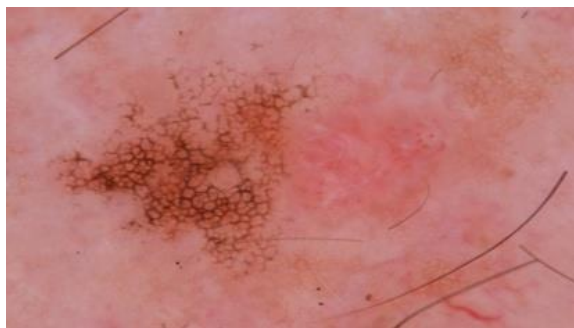

Fig. 6. Vascular Lesions

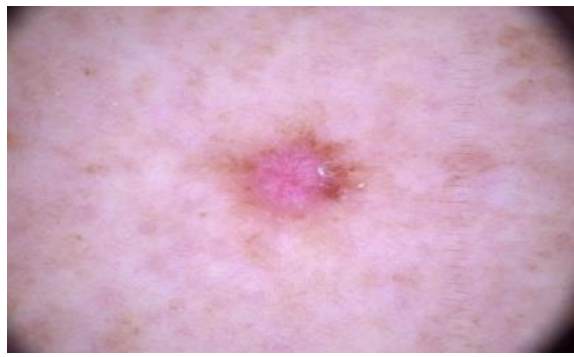

Fig. 7. Actinic keratosis

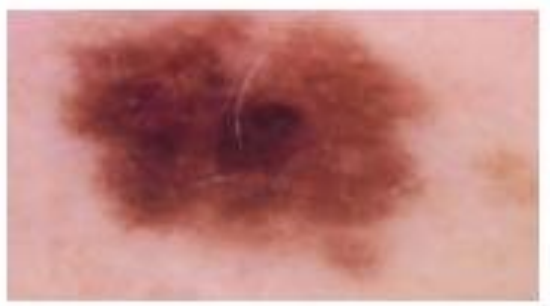

Fig. 8. Dermatofibroma

\section{TYPES OF SKIN CANCER}

\section{A. Basal Cell Carcinoma}

Basal cell carcinoma is a cancer that creates on parts of your skin that get a lot of sun. This cancer is most likely not going to spread from your skin to various bits of your body, anyway it can move close by into bone or other tissue under your skin. A couple of meds can shield that from happening and discard the cancer. The tumors start off as meager sparkly thumps, as a general rule on your nose or various bits of your face. Regardless, you can get them on any bit of your body, including your trunk, legs, and arms.

\section{B. Benign-keratosis-Lesion}

Benign-keratosis-Lesion are the most broadly perceived benign cutaneous tumors, and in adults Benign-keratosisLesion skin are warty, keratotic skin advancement that first present after the fourth decade. The measure from two or three millimeters to centimeters the concealing extents from pink to tan to dull dim shaded. Lesions can be particular or different. After some time, patients develop wherever from a couple to a few Benign-keratosis-Lesion. Various patients request removal of Benign-keratosis-Lesion skin, particularly when different or enormous, considering their ugly appearance.

\section{Dermatofibroma}

Dermatofibroma is a regular cutaneous handle of obscure etiology that happens considerably more typically in ladies. Dermatofibroma an incredible piece of the time makes on the farthest centers (by and large the lower legs) and is regularly asymptomatic, despite the manner in which that pruritus and delicacy can be open. It is amazingly the most by and large saw inconvenient skin tumor. Diverse well-portrayed, histologic subtypes of dermatofibroma have been spoken to. Clearing of the tumor is generally not usually required except for if definitive shortcoming exists or especially upsetting manifestations are available.

\section{Melanocytic Nevi}

Melanocytic nevi are liberal neoplasms of melanocytes and appear in a ton of varieties, which all are joined into our game-plan. The groupings may isolate in a general sense from a dermatoscopic point of view. As opposed to melanoma they are commonly symmetric concerning the disseminating of masking and structure.

\section{E. Melanoma}

Melanoma is a malignant neoplasm gotten from melanocytes that may show up in various assortments. At whatever point isolated in a beginning time it will when all is said in done be soothed by direct wary extraction. Melanomas can be noticeable or noninvasive. We combined all assortments of melanoma solidifying melanoma, in any case banned non-pigmented, subungual, visual or Melanomas.

\section{F. Vascular}

Vascular lesions are generally run of the mill assortments from the standard of the skin and basic tissues, significantly more reliably known as pigmentations. There are three basic classes of vascular lesions: Hemangiomas, Vascular Malformations, and Pyogenic Granulomas. While these skin pigmentations can seem, by all accounts, to be close now and again, they each change like starting stage and essential treatment.

\section{RESULT}

In this csv file, there are 6 independent features and one target variable.

Independent Features - lesion id, image id, dx_type, age, sex, localization.

\section{Dependent variable: $\mathrm{dx}$}

Lesion id and image id is inserted based on image name and used in the dataset. Localization feature in the dataset determines the position of skin where the possibility of skin cancer is detected and further the procedure of classification will be done based on all the independent variables and images given as the input.

Published By:

Blue Eyes Intelligence Engineering \& Sciences Publication

(C) Copyright: All rights reserved. 
Skin Cancer Classification using Random Forest

\begin{tabular}{|c|c|c|c|c|c|}
\hline 1 & lesion_id image_id $\mathrm{dx}$ & dx_type & age & \multicolumn{2}{|c|}{ localization } \\
\hline 2 & HAM_000CISIC_0027. bkJ & histo & 80 male & scalp & \\
\hline 3 & HAM_000C ISIC_0025i bkl & histo & 80 male & scalp & \\
\hline 4 & HAM_000: ISIC_0026 bkl & histo & 80 male & scalp & \\
\hline 5 & HAM_000: ISIC_0025i bkl & histo & 80 male & scalp & \\
\hline 6 & HAM_000:ISIC_0031ibk1 & histo & 75 male & ear & \\
\hline 7 & HAM_000: $151 C$ 0027: bkl & histo & 75 male & ear & \\
\hline 8 & HAM_000: ISIC_0029: bkJ & histo & 60 male & face & \\
\hline 9 & HAM_000: ISIC_0029r bkJ & histo & 60 male & face & \\
\hline 10 & HAM_000: ISIC_0025: bkl & histo & 70 female & back & \\
\hline 11 & HAM_000: $15 \mid \mathrm{C} \_0025$; bkl & histo & 70 female & back & \\
\hline 12 & HAM_000: ISIC_0025: bkd & histo & 55 female & trunk & \\
\hline 13 & HAM_000: ISIC_0029: bkJ & histo & 85 female & chest & \\
\hline 14 & HAM_000 ISIC_0025! bkl & histo & 85 female & chest & \\
\hline 15 & 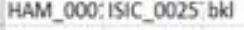 & histo & 70 male & trunk & \\
\hline 16 & HAM_000: 15iC_0032. bkj & histo & 70 male & trunk & \\
\hline 17 & HAM_000:ISIC_0031:bkl & histo & 65 male & back & \\
\hline 18 & HAM_000:ISIC_0025! bkl & histo & 75 male & \multicolumn{2}{|c|}{ upper extremity } \\
\hline 19 & HAM_000: ISIC_0031i blj & histo & 75 male & \multicolumn{2}{|c|}{ upper extremity } \\
\hline 20 & HAM_000:15iC_0029: bkJ & histo & 70 male & chest & \\
\hline 21 & HAM_000:15iC_0032: bkl & histo & 70 male & chest & \\
\hline 22 & HAM_000t ISIC_0032. blkJ & histo & 70 female & face & \\
\hline 23 & HAM_000: ISIC_0025ibkl & histo & 60 male & back & \\
\hline
\end{tabular}

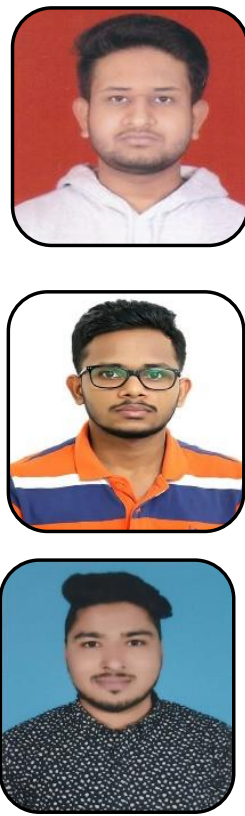

Mohammed Abdul Sofiyan, $3^{\text {rd }}$ year cse undergraduate pursuing B.tech in SRM Institute of science and technology.

\section{Fig. 9. TRAINING DATA SET}

\section{CONCLUSION}

In this paper we have discussed about the classification of 7 different types of skin lesions using step by step algorithm. We have used Random Forest Algorithm to make decision about the type of diagnosis. And overall, we have got the accuracy of $97.3 \%$ on training dataset. We hope that our work will be beneficial for any person researching on this field to make further advancement.

\section{REFERENCES}

1. Argenziano, G. et al. Interactive Atlas of Dermoscopy (Edra Medical Publishing and New Media: Milan, 2000).

2. Esteva, A. et al. Dermatologist-level classification of skin cancer with deep neural networks. Nature 542, 115-118 (2017).

3. Stevenson, A. D., Mickan, S., Mallett, S. \& Ayya, M. Systematic review of diagnostic accuracy of reflectance confocal microscopy for melanoma diagnosis in patients with clinically equivocal skin lesions. Dermatol Pract Concept 3, 19-27 (2013).

4. Akay, B. N., Kocyigit, P., Heper, A. O. \& Erdem, C. Dermatoscopy of flat pigmented facial lesions: diagnostic challenge between pigmented actinic keratosis and lentigo maligna. Br J Dermatol 163, 1212-1217 (2010).

5. Tschandl, P., Rosendahl, C. \& Kittler, H. Dermatoscopy of flat pigmented facial lesions. J Eur Acad Dermatol Venereol 29, 120-127 (2015).

6. Lallas, A. et al. The dermatoscopic universe of basal cell carcinoma Dermatol Pract Concept 4, 11-24 (2014).

7. Braun, R. P. et al. Dermoscopy of pigmented seborrheic keratosis: a morphological study. Arch Dermatol 138, 1556-1560 (2002).

8. Zaballos, P. et al. Dermoscopy of solitary angiokeratomas: a morphological study. Arch Dermatol 143, 318-325 (2007).

9. Zaballos, P. et al. Dermoscopy of pyogenic granuloma: a morphological study. Br J Dermatol 163, 1229-1237 (2010).

10. Wurm, E. et al. The value of reflectance confocal microscopy in diagnosis of flat pigmented facial lesions: a prospective study. J Eur Acad Dermatol Venereol 31, 1349-1354 (2017).

\section{AUTHORS PROFILE}

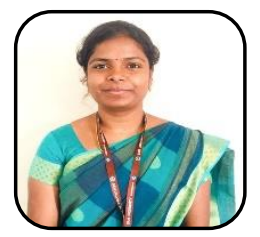

MS. S. Nandhini, Assistant Professor (O.G) at SRM Institute of science and technology. Work experience in area of Image Processing.

Sushant Kumar, $3^{\text {rd }}$ year cse undergraduate pursuing B.tech in SRM Institute of science and technology.

Adnan Afridi, ${ }^{\text {rd }}$ year cse undergraduate pursuing B.tech in SRM Institute of science and technology. 